\title{
CarAgent: Collecting and disseminating floating car data in vehicular networks
}

\author{
Hui Huang ${ }^{\mathrm{a}, \mathrm{b}, *}$, Mahbub Hassan ${ }^{\mathrm{a}, \mathrm{b}}$, Glenn Geers ${ }^{\mathrm{a}, \mathrm{c}}$, Lavy Libman ${ }^{\mathrm{d}}$ \\ a School of Computer Science and Engineering, University of New South Wales, NSW 2052, Australia \\ b Data61, CSIRO, 13 Garden St, Eveleigh, NSW 2015, Australia \\ c ARRB Group Ltd, 2-14 Mountain St Ultimo, NSW 2007, Australia \\ d Google Australia, 48 Pirrama Rd, Pyrmont, NSW 2009, Australia
}

\section{A R T I C L E I N F O}

\section{Article history:}

Received 19 January 2018

Received in revised form 16 July 2018

Accepted 9 August 2018

Available online 13 August 2018

\section{Keywords:}

Intelligent transportation systems

Floating car data

V2V communication

Heterogeneous vehicular networks

\begin{abstract}
A B S T R A C T
Floating car data (FCD) refers to the motion and sensor data produced by moving vehicles on the road. Given that traffic management authorities and drivers can benefit from FCD enormously, there is an urgency to develop efficient FCD collection and dissemination techniques that scale with peak road traffic. In this paper, we present CarAgent, a message exchange protocol that periodically collects and uploads FCD to data centres with the minimal utilisation of existing mobile network resources. We also show how CarAgent can be easily extended to efficiently disseminate FCD to all vehicles in a target area using Dedicated Short Range Communication (DSRC), a recently released wireless communication standard for vehicles. We analytically derive the key performance metrics of CarAgent and validate them with simulations. We evaluate CarAgent using microscopic simulation of road traffic in real street maps while incorporating wireless protocol details of Long Term Evolution (LTE) and DSRC. Simulation results confirm that, compared to the state-of-the-art, CarAgent consumes $50 \%$ less LTE resources for FCD collection. For FCD dissemination, CarAgent consumes 45\% less DSRC resources while improving the speed of dissemination significantly.
\end{abstract}

(C) 2018 Elsevier Inc. All rights reserved.

\section{Introduction}

Modern vehicles continuously generate a wide range of motion, position, and sensor data, which are often referred as floating car data (FCD) [1,2]. FCD can be valuable for monitoring road traffic in real time and explore solutions for future expansion of road infrastructures and services [3-5]. It is therefore important to collect FCD from the vehicles on the road and upload them periodically to data centres equipped with advanced data analytics and backup facilities. Some FCD aggregates, such as the average vehicle speed of different road segments in a target area, can aid real-time navigation for motorists. Fast and efficient dissemination of such FCD aggregates to all vehicles in a target area, therefore, is also desirable.

The realisation of FCD collection and dissemination will require the development of appropriate wireless communication standards

\footnotetext{
* Corresponding author at: School of Computer Science and Engineering, University of New South Wales, NSW 2052, Australia.

E-mail addresses: hui.huang@unsw.edu.au (H. Huang), mahbub.hassan@unsw.edu.au (M. Hassan), glenn.geers@arrb.com.au (G. Geers), libman@google.com (L. Libman).
}

for vehicles. In this regard, two noteworthy developments have taken place in recent years. In 2010, a new wireless communication standard, called DSRC [6], has been released to enable vehicles to communicate and exchange data directly with other nearby vehicles. Design of DSRC, however, was motivated by the road safety. To help prevent accidents, DSRC dictates that every vehicle periodically ( 1 to 10 times per second) broadcasts its travelling status in small beacon messages called Cooperative Awareness Messages (CAMs) [7]. DSRC also has the provision for vehicles to upload data to data centres via special DSRC road side units (RSUs). Unfortunately, due to the huge cost of RSU deployments, DSRC RSUs are yet to be widely implemented.

Recently, Long Term Evolution (LTE), the standard platform underpinning the 4th and 5th generation mobile networks, has become popular with the vehicle manufacturers for directly connecting individual vehicle to the Internet [8]. With LTE connectivity, a vehicle no longer requires a DSRC RSU to upload data. Instead, they can upload FCD to a remote data centre using the same LTE mobile network that we use to connect our smartphones. This development is motivating researchers to consider simultaneous use of both LTE and DSRC for FCD collection and dissemination $[2,9,10]$. Although LTE and DSRC together provide a promising platform for collecting and disseminating FCD, scalability remains a major issue. 


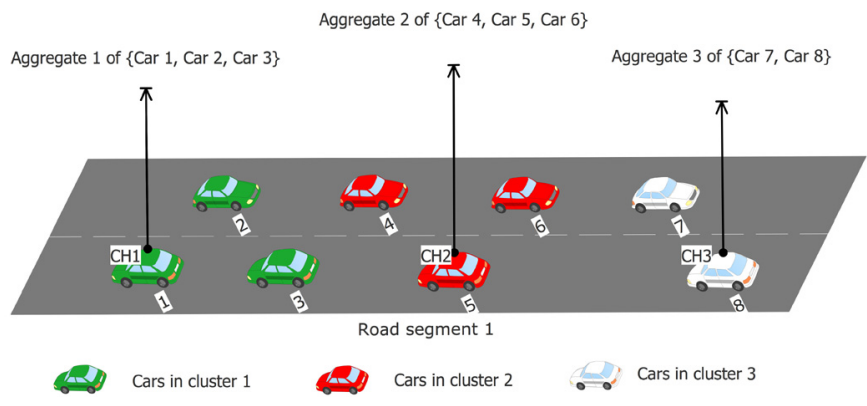

Fig. 1. Cluster based FCD collection.

One of the main challenges of FCD collection and dissemination is the extremely high load created on the limited radio resources of LTE and DSRC when a large number of vehicles attempt communication during peak traffic.

The state-of-the-art solution to the LTE overload problem is to use the cooperative awareness enabled by DSRC to create clusters of vehicles having common features (e.g. proximity, travel direction, speed, connectivity) [11-13], and let a single vehicle from the cluster, i.e., the cluster head, upload the aggregated statistics from all cluster members. Cluster-based data aggregation and collection do improve the situation from individual collections, but they are not very efficient when aggregate statistics for each road segment are to be collected, which is typically required by most data centre analytics [14,15].

The main source of inefficiency of cluster-based approach arises from the fact that vehicles on a given road segment, even they are located in close proximity, could be divided into many separate clusters, as illustrated by the example in Fig. 1. In this example, the 8 vehicles are grouped into 3 clusters, where each cluster head will upload an aggregate for the road segment to the server, resulting in a total of three uploads for the same road segment. The main reason for the road segment fragment is because of the lossy nature of DSRC channel: as vehicles need to exchange control messages with each others to form clusters, loss of these messages result in the cluster overlapping problem [16], which produces more number of clusters in small range, hence reducing the FCD collection efficiency. The increased load on the network can worsen the situation, i.e., the number of different clusters formed increases with increasing number of vehicles (evidence from microscopic road traffic simulation is provided in Section 5).

The scalability problem also arises with DSRC utilisation if vehicles attempt to disseminate FCD to all vehicles in a target area through vehicle-to-vehicle (V2V) communications. The state-ofthe-art FCD dissemination methods [17-19] require each vehicle to periodically broadcast selected data from its local database to other vehicles in the vicinity. Upon hearing the broadcast, other vehicles update their own local databases. However, periodic dissemination can dramatically increase communication overhead in dense traffic threatening the collapse of DSRC services. A detailed simulation study revealed [20] that, with periodic broadcasting of 300-byte FCD messages once every second, DSRC would be fully saturated when traffic density reaches to 22 vehs/km/lane, which is below peak-hour traffic in many cities. Although conceptually it is possible to reduce DSRC load by increasing the broadcast interval during peak hours, studies have shown that FCD dissemination latency increases exponentially with a linear increase in broadcast interval [21]. Since FCD dissemination aims to aid real-time navigation, large broadcast intervals are not desirable, which leaves the efficient FCD dissemination over DSRC as an open research problem.

In this paper, we present CarAgent to address the aforementioned problems in FCD collection and dissemination. The proposed approach employs a set of FCD collectors called agents, which are protocol packets capable of moving from one host to another in the network, to collect and disseminate FCD among the target region. Our idea is similar to the concept of Mobile Agents (MA), which has been explored for data collection in wireless sensor networks $[22,23]$ and routing path discovery in mobile ad hoc networks (MANETs) [24-26]. We believe that our approach can provide a flexible and effective solution to FCD collection and dissemination, as the agents can migrate from one vehicle to another at the speed of wireless communications and collect road segment aggregates effortlessly without having to constantly form clusters and select cluster heads. Besides, since different data types and applications require different data fusion algorithm (e.g. how to merge the collected individual data into a single aggregate), the local data processing could be easily re-programmed by simply modifying the execution code carried by agents, enabling easy re-tasking on the vehicular network.

While collecting FCDs and migrating through the network, agents can also serve as a natural platform to disseminate the same data to other vehicles in the target area. Thus, the CarAgent protocol has the potential to serve as both FCD collector and disseminator, which further simplifies deployment efforts and complexity.

Our contributions can be summarised as follows:

- We propose a novel FCD collection and dissemination framework, called CarAgent, which combines the services of both LTE and DSRC. To the best of our knowledge, this is the first attempt to apply agent concept to FCD collection and dissemination in complex urban road networks.

- We analytically derive the key performance dynamics of CarAgent and validate them through extensive simulations.

- We compare the performance of CarAgent against state-of-theart using microscopic simulations of road traffic in real street maps of different road patterns while incorporating wireless protocol details of both LTE and DSRC. Simulation results confirm that, compared to the state-of-the-art, CarAgent reduces wireless network resource consumptions by $50 \%$ and $45 \%$ for LTE and DSRC, respectively, while improving the speed of FCD dissemination over DSRC significantly.

The rest of this paper is organised as follows. Section 2 describes related works. Section 3 presents the system model of CarAgent followed by its analytical modelling and numeric study in Section 4. Performance evaluation based on microscopic road traffic simulation is presented in Section 5. We draw conclusions in Section 6.

\section{Background and related works}

\subsection{Mobile agent in ad hoc networks}

Mobile agent based information collection and dissemination has been well studied in the context of Wireless Sensor Networks (WSN) [22,23]. These approaches do not require sensor nodes to directly send raw data to the data sink, on the other hand, the sink node creates mobile agents, which migrate among the network to collect reliable data. The problem, therefore, can be reduced to a variant of the NP-hard Travelling Salesman Problem (TSP) such that the agent is required to find the shortest possible route that visits each node exactly once and returns to the data sink in the graph induced by the network topology. However, the data collection schemes proposed for WSNs do not map well to vehicular networks due to the dynamic network topology caused by high node mobility.

In the context of Mobile Ad Hoc Networks (MANETs), many works on using mobile agents focus on the routing aspect [24,26]. 
Due to the dynamic nature of MANETs, route discovery and maintenance is a quite challenging task. It has been found that Ant Colony Optimisation (ACO) algorithms [27], which deploys multiple simple agents called artificial ants to establish optimum paths between source and destination by mean of stigmergy, can give better results as they are having the characterisation of swarm intelligence, which is highly suitable for such type of volatile network.

Concerning the use of mobile agent for information dissemination in VANETs, only a few works were established in recent years for the purpose of service discovery and data collections. In [28], the authors proposed the ADOPEL protocol using the mobile agent to collect data for delay non-sensitive applications based on Reinforcement Learning (RL), the mobile agent can adaptively choose the next relay nodes along the road segment that offers a better chance to collect more data without losing the way back to the data sink. However, this work only considers the case of the single agent in a simplified highway scenario, and the approach might be inadequate to address the same problem in the urban area due to the complicated road network topology. In [29], a mobile agent-based scheme for service discovery in MANETs is proposed, where mobile agents are travelling through the network, collecting as well as delivering the dynamically changing service information. Their scheme is further modified and extended in [30] to be suitable for the context of VANETs, such that mobile agents migrate based on the moving speed, direction and location of vehicles to disseminate information to others of the broader area in a shorter time. However, their works only focus on the one-to-many dissemination scenario, where mobile agents are created by an entity who wants to broadcast a piece of information to vehicles within a given region.

\subsection{Traffic information system in vehicular networks}

Several works have been recently conducted to investigate the non-safety related FCD collection and dissemination issues by adopting different techniques and approaches. In the case of FCD collection, the recent trend has focused on FCD collection in heterogeneous networks, within which vehicles possess two interfaces: 1) 802.11p/DSRC and 2) LTE at the same time [31]. A common paradigm is to use the cooperative awareness enabled by DSRC to create clusters of vehicles having common features (e.g. proximity, travel direction, speed, connectivity). After the cluster formation, each Cluster Head $(\mathrm{CH})$ collects data from their cluster members, and then the aggregated information is delivered to the FCD server on the cloud. For example, in [12], the authors proposed the LTE4V2X to collect the data from vehicles on the road. Their work utilises eNodeBs to organise vehicles into several clusters. The selected $\mathrm{CHs}$ then can collect data from cluster members using 802.11p/DSRC communication and then upload the aggregated information to the server via eNodeBs. In their later extension paper [13], multi-hop communication technique is applied to collect data inside areas where there is no LTE coverage (e.g. tunnel).

In general, the data collection protocol proposed in the abovementioned works requires to form and maintain some certain structures among vehicles. In a highly dynamic vehicular network, vehicles join and leave clusters along their travel route, causing changes in the cluster structure. Frequent changes in cluster membership, splitting and merging of clusters increase the control overhead and drain the radio resources [16]. In order to overcome this problem, more recently, the author in [32] proposed an FCD collection approach based on the On-the-Fly Clustering (OFC) technique. In their work, vehicles are assumed to know the time instance when the collection starts. Upon collection interval starting, every vehicle computes its own sending timer based on the current LTE channel quality and the number of one hop DSRC neighbours.

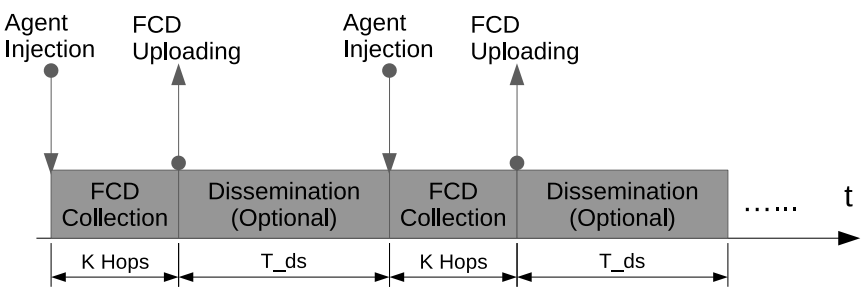

Fig. 2. Overall operation of the CarAgent system.

Vehicles whose timer expire become cluster heads and upload the aggregated data of their one-hop neighbours to the server. Immediately after the uploading, a cluster head broadcasts an Inhibit message over the DSRC network to suppress other vehicles from uploading duplicated information. However, since they assume vehicles need to know the starting time instance for each collection cycle, their approach cannot cope with on-demand collections. In addition, the OFC algorithm does not take the road network topology into consideration, a road segment might be covered by several different clusters, results in several fragments thus lessoptimised data aggregation efficiency for collecting road segment aggregates.

As for the traffic information dissemination, current approaches reported in the literature usually follow the push-based model, or periodic dissemination model [33,34], where each vehicle periodically select a set of sensor readings or important messages, and then broadcast them to its vicinity. Other vehicles upon reception then rebroadcast the received information based on certain policies and criterion.

Since periodic dissemination can dramatically increase communication overhead when the traffic density becomes high [14], network performance would be severely affected, leading to bandwidth waste. In order to overcome this deficiency in the pushbased model, some studies suggested to adaptively adjust the frequency of periodic broadcasting based on message utility and channel quality. For example, the author in $[18,19]$ proposed the Adaptive Traffic Beacon (ATB) protocol to exchange traffic information among vehicles on the road. The broadcast interval is adaptively adjusted based on the channel quality indicator calculated by considering the number of packet collisions, Signal to Noise (SNR) level and the number of neighbours. The goal of ATB is to send as many information as possible, but avoid overloading the wireless channel at any time. Their simulation results indeed show that the increasing rate of communication overhead as a function of traffic density can be effectively reduced, however, the trade-off being made is longer dissemination latency due to the increased broadcast interval [21].

\section{System model}

The overall operation of the CarAgent is illustrated in Fig. 2. To collect FCD from a given target area, a cloud server periodically injects a batch of mobile agents into the VANET covering the area using LTE. The agents collect FCD aggregates from the VANET using DSRC and report them back to the server via LTE. After reporting the aggregates to the server, they can optionally roam inside the VANET for some time to disseminate the collected information to the vehicles using DSRC. Agents eventually self-terminate themselves after a predetermined lifetime. In the following, we describe the system models for roads and vehicles, and the agents for FCD collection and dissemination.

\subsection{Roads and vehicles}

The road network is divided into many road segments, where a road segment is defined as the road span from one intersection 
to the next intersection. If the distance between two intersections is larger than the effective DSRC communication range (e.g. urban highway), the road span should be further divided into smaller ones so that vehicles located within the same road segment are able to communicate with each other directly. We also assume that each vehicle is equipped with a GPS receiver and digital maps for obtaining the road segmentation scheme, location and time information. The GPS positioning accuracy is assumed to be precise enough for the vehicles to locate themselves in specific road segments. In contrast, the server cannot locate vehicles within road segments, but it can determine whether a vehicle is inside a target area or not, thanks to the LTE tracking capability using base stations.

Vehicles equipped with both DSRC and LTE, move through the road network in a target geographical area. Vehicles use DSRC for V2V communications and LTE for communicating with the cloud server. The proposed CarAgent, instead of harvesting individual FCD, collects FCD aggregates describing road segment performance. Following the definition in [14], an FCD aggregate is a tuple that contains information about a specific road segment, as the following:

$A:=(\mathcal{S}, \mathcal{T}, v, \mathcal{Q})$

where $\mathcal{S}$ is the road segment's ID, $\mathcal{T}$ is the time stamp and $v$ is the primary value of the aggregate, which conveys values of FCD produced by vehicles in this road segment, such as speed, heading, road conditions and road occupancy. The item $\mathcal{Q}$ is the auxiliary value used by many aggregation schemes to denote the certainty or quality of information after it has been aggregated, i.e. standard deviation of an average or count of FCDs summarised in the aggregate.

It is assumed that each vehicle locally stores the FCD from CAM broadcasts it receives from other vehicles. Therefore, a vehicle can compute an FCD aggregate of the road segment it stays when needed, given that the road segmentation scheme is known in advance. How to merge individual FCD into an aggregate is out of our scope, in fact, it most depends on the type of data and the particular applications. For example, geographical coordinates might be fused by calculating their bounding box and the average and minimum speed could be used for traffic congestion monitoring. This design decision can reduce the amount of data being transmitted to the remote server. More importantly, it could improve data privacy as individual data usually "disappear" into the aggregates [14].

\subsection{CarAgent: FCD collection}

In this section, we explain how CarAgent collects FCD aggregates from the network. In particular, we describe the agent format, agent injection into VANET, and hopping of agents within VANET.

\subsubsection{Agent format}

In this context, the agent is a data packet whose format is shown in Fig. 3. As mentioned before, the cloud server periodically inject a batch of agents into the target region, agents injected from the same batch has the same batch ID, and the agent ID field uniquely identifies each agent within the batch. Since how to merge individual FCDs into an FCD aggregate depends on the data of interest as well as the particular application (e.g. lossless/lossy fusion, average/maximum calculation), each agent also carries a piece of execution code to determines the local data processing in each hop. Clearly, the use of agent provides high flexibility of the FCD collection process. By changing the execution code of agents,

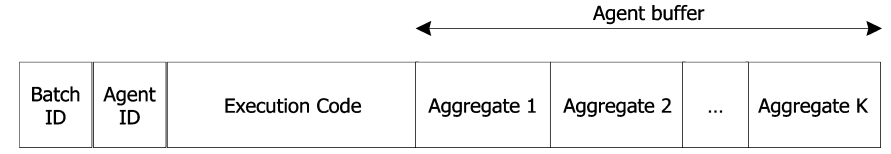

Fig. 3. Agent format.

different data processing and aggregation can be carried out at each hop.

Beside, the agent also maintains a buffer that can store up to $K$ collected FCD aggregates. The value of $K$ is limited to ensure that the maximum length of an agent can be transported within a single DSRC MAC frame to avoid packet fragmentation, which would increase system complexity and reduce the reliability of agent hopping.

\subsubsection{Agent injection}

Because a single agent can only collect FCD aggregates from a small number of road segments due to the limited buffer, to cover a large target area, a server is therefore required to inject a batch of $N$ agents randomly to $N$ distinct vehicles within the target area each time it wants to learn the current traffic status of the area.

To achieve this, we assume a special-purpose backend server as foreseen in the ETSI specification [35] for supporting geocasting services in LTE-based vehicular networks. The backend server maintains a list of geographical areas. Vehicles crossing over a new area register themselves, allowing the server to know their present in any area at all times and their IP addresses. Note that, the assumption of the existence of such a backend server does not undermine the usefulness of the proposed CarAgent system, as the server does not store precious location information of each vehicle, but only be able to determine whether a vehicle is inside a target area or not

\subsubsection{Agent hopping algorithm}

Once an agent enters (injected) into the VANET, it keeps hopping from one vehicle to another until $K$ hops, collecting and buffering FCD aggregates for distinct road segments along its path. Each agent therefore will store at most $K$ FCD aggregates, where the parameter $K$ is designed to ensure that the agent can be contained within a single DSRC MAC frame.

Recall that each vehicle locally stores the individual FCDs by processing the periodic CAMs broadcast by DSRC. Once an agent hops to a vehicle located at road segment $i$, the agent then can get access to the local storage of the host vehicle, run the execution code to create an FCD aggregate of that road segment. Note that due to channel fading and packet collisions, the host vehicle might not be able to receive the CAMs from all vehicles within the same road segment. In this sense, the CarAgent can only achieve the "best effort" FCD aggregations. As suggested by [14,15], most traffic efficiency applications can tolerate a certain loss of information.

All that is required is for the agent to successfully hop from one road segment to another segment. Therefore, we propose a 4-way handshake algorithm to help the agent successfully hop to the next road segment randomly. The algorithm is described next:

- The vehicle currently hosting the agent broadcasts the agent and sets a timer, $t_{\text {wait }}$, waiting for replies from potential vehicles from other road segments to host the agent.

- Upon receiving an agent, a vehicle first checks whether it is located in the same road segment from where the agent was broadcast. If the vehicle is on the same road segment, it ignores the agent. Otherwise, it temporarily stores the agent and sets a timer, $t_{\text {reply }}$, randomly drawn from the interval of $\left(0, t_{\text {wait }}\right]$. 


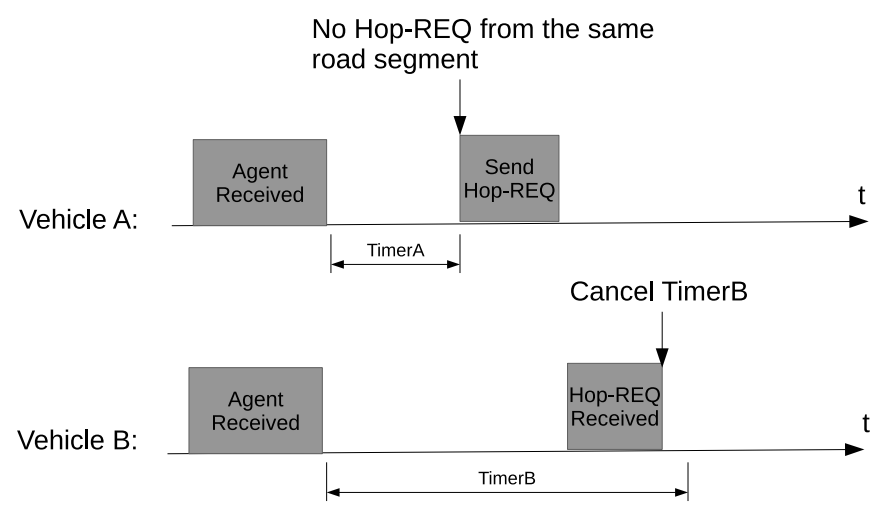

Fig. 4. Time domain illustration of next forwarder selections.

- Once the timer $t_{r e p l y}$ expires, the vehicle temporarily storing the agent replies with a broadcast Hop-REQ message. Another vehicle, located on the same road segment, cancels its own $t_{\text {reply }}$ if it overhears a Hop-REQ before its timer $\left(t_{\text {reply }}\right)$ expires. In other words, only one vehicle from each road segment is expected to reply with Hop-REQ.

- The current host vehicle temporarily stores all received Hop$R E Q s$ before its timer $t_{\text {wait }}$ expires. Upon expiry of $t_{\text {wait }}$, the host vehicle restarts the broadcast process if it does not receive any Hop-REQ. Otherwise, it chooses the next vehicle from a road segment not visited before if there is one, otherwise randomly chooses one among the received Hop-REQ as the next holder and unicasts a Hop-CFM to it.

- If the original agent holder does not receive the MAC layer ACK from the next holder, it restarts the migration process to prevent agents from unexpected drops.

Fig. 4 shows the time domain illustration of the next forwarder selections of the proposed agent migration algorithm. Consider a scenario in which two vehicles, $A$ and $B$, located at the same road segment receive an agent broadcast from a neighbouring road segment. Vehicle $A$ does not overhear any Hop-REQ from other vehicles located on the same road segment, it then sends a Hop-REQ after TimerA expires. Vehicle $B$, during its timer counting down, overhears the Hop-REQ from $A$. It then stops the contention process.

Note that the proposed 4-way handshake algorithm uses unicast of Hop-CFM with MAC layer acknowledgement, which might lead to the head of line blocking effect in highly dynamic vehicular environment [37]. However, simply using a broadcast message to confirm the next agent holder could result in unexpected agent drops if the Hop-CFM is lost. The trade-off being made here is the improvement of the reliability of agent migration with the price of the possible additional delays on messages waiting on the queue. Considered that the proposed CarAgent only introduce small traffic over the DSRC channel (evidence from microscopic road traffic simulation is provided in Section 5), the overhead caused by the MAC layer ARQ process is very small. Moreover, 802.11p allows the use of different access categories (AC), the impact of blocked queue on safety application could be relieved by assigning lower priority to the Hop-REQ messages.

It is clear that the timer $t_{\text {wait }}$ dictates the hopping latency of each agent. Therefore, a smaller $t_{\text {wait }}$ would lead to faster agent hopping, reducing latency for FCD collection. However, as studied in [38], too small timer leads to broadcast overload problem in contention-based networks. The optimal setting of $t_{\text {wait }}$ depends on the congestion control mechanism as well as the current channel loads. Following the recommendations in $[36,39]$, we choose $t_{\text {wait }}=25 \mathrm{~ms}$.

\subsubsection{FCD uploading}

After $K$ hops, agents stop collecting new FCD and upload the collected aggregates to the server. A naive approach for the agents would be to simply upload every buffered aggregate to the server However, this would result in redundant uploading as the same road segment could be sampled by more than one agents. In order to prevent duplicate uploads, CarAgent implements the following simple scheme. Each vehicle maintains a variable, which is initially set to -1 , indicating that the road segment that the vehicle is currently staying at has not been sampled by any agent during the current collection cycle. The variable is updated under the following rules:

- If the vehicle overhears an agent broadcast from another vehicle located at the same road segment, it sets the variable to the agent's batch ID, indicating that the road segment now has been sampled.

- Every time a vehicle moves to a new road segment, it resets the variable to -1 .

An agent samples the current road segment only if the variable of the current holder does not equal to it's batch ID. After $K$ hops, the agent simply uploads every buffered aggregates to the server by using LTE uplink. The approach is feasible as agent hopping takes only a few milliseconds, and each agent only hops $K$ times ( $K$ is usually a small value), during which vehicles move only a very small distance. As we will show in Section 5 , the proposed scheme significantly reduces redundant uploads for CarAgent.

\subsection{CarAgent: $F C D$ dissemination}

After the data collection and upload (after $K$ hops), the batch of agents can be treated as a distributed floating database containing traffic information of road segments of the target area. Therefore, by simply prolonging the lifetime of agents by an additional time interval, $T_{d s}$, it is possible to disseminate the collected FCD aggregates to other vehicles in the area using the free DSRC spectrum. This can be achieved by letting the agents continue to hop around the VANET and allowing other vehicles to accumulate the FCD aggregates as they opportunistically overhear the agent broadcasts. Over time, each vehicle then will learn the traffic condition of the target area. Longer the agents hop around, the more opportunities the vehicles get to build the knowledge. In Section 4, we will analytically model the speed of dissemination in terms of learning the fraction of the target area as a function of time.

During dissemination, agents do not collect any new FCD aggregates, but they aim to deliver the collected information to as many vehicles in the target area as possible. To roam the entire target area as rapidly as possible, the agents, therefore, need to hop as far as possible. Inspired by the ETSI GeoNetworking standard [40], the new hopping objective, i.e., extending the hop distance as far as possible at each hoping attempt, can be achieved by modifying the timer setting criterion of $t_{\text {reply }}$ as follows:

$t_{\text {reply }}= \begin{cases}t_{\text {wait }}+\frac{t_{\text {min }}-t_{\text {wait }}}{R_{c}} D & D \leq R_{C} \\ t_{\text {min }} & D>R_{C}\end{cases}$

where $R_{C}$ is the effective DSRC communication range and $D$ denotes the distance between the recipient vehicle and the current agent holder. The $t_{\min }$ is set to $1 \mathrm{~ms}$ following the default setting of the ETSI GeoNetworking standard.

\section{Performance modelling}

To evaluate the performance of the proposed CarAgent system, we define the following two performance metrics: 


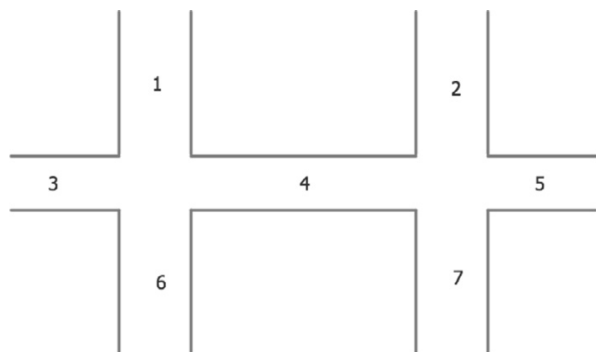

(a)

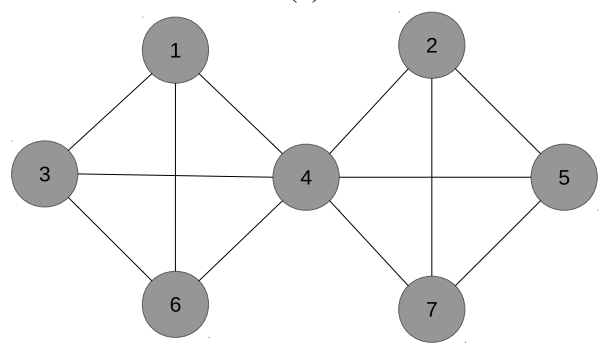

(b)

Fig. 5. (a) An urban road network, and (b) the corresponding undirected graph.

- Expected Collection Coverage $\Gamma(N, K)$ : This metric refers to the expected fraction of all road segments for which the server is able to collect the FCD after injecting and receiving a batch of $N$ agents, where each agent completes $K$ hops before uploading the collected data. The higher the fraction, the better the coverage, and vice versa.

- Dissemination Speed $\Omega(t)$ : For a given vehicle in the target area, we define vehicle awareness as the fraction of road segments it learns about by overhearing agent broadcasts over DSRC. The dissemination speed is then expressed as the expected vehicle awareness as a function of time spent by agents roaming in the VANET after their initial $K$ hops of FCD collection.

In this section, we derive these two metrics analytically and study their properties with numerical experiments.

\subsection{Derivation of $\Gamma(N, K)$}

A road network can be represented by an undirected graph, $G(V, E)$, where $V$ is the set containing every road segments, and an edge $e_{i j} \in E$ if nodes $i$ and $j$ are connected by an intersection. Fig. 5 illustrates the graph representation of a typical urban grid road network.

As agents can only hop from one road segment to any of its neighbour road segments, the most basic hopping behaviour of an agent can be modelled as a random walk on graph $G$. With random walk, an agent staying at road segment $i$ will hop to segment $j$ with probability $p_{i, j}=\frac{1}{d(i)}$ if $e_{i j} \in E$, where $d_{i}$ is the degree of node $i$. Obviously, the random walk model will accurately capture the hopping of agents if, at the time of agent broadcast, every neighbour road segment has at least one vehicle within the radio coverage of the broadcast. Otherwise, the condition $p_{i, j}=\frac{1}{d(i)}$ does not hold, causing the random walk model overestimate the performance of CarAgent.

Definition 1. If at the time of agent broadcast, every neighbour road segment has at least one vehicle within the radio coverage of the broadcast, the vehicular traffic in the road network is called saturated traffic.
Clearly, the random walk model will provide an upper bound for CarAgent performance, which can be achieved with saturated traffic. In Section 5, using simulations, we will identify the minimum vehicle density required to satisfy the saturated traffic condition for any road network. Interestingly, simulations reveal that even a light traffic with approximately 20 vehicles per kilometre on average can achieve the saturated traffic status for the purposes of random walk. Our definition of saturated traffic therefore by no means indicate heavy or peak traffic, which is expected only during certain hours of the day.

Assuming the random walk is ergodic and irreducible, there exists a steady state probability that the walker will visit node $i$ with probability $\pi_{i}=\frac{d_{i}}{2|E|}$ [41]. This also means that if an agent was injected or initially placed into the area following the steady state distribution, then the probability that it would visit road segment $i$ in the next hop would also be $\pi_{i}$. Based on this, we have:

Lemma 1. Under the assumption of saturated traffic and steady state agent injections, for any arbitrary road network, $\Gamma(N, K)=$ $\frac{\sum_{i \in V} 1-\left(1-\frac{d_{i}}{2 \mid E)^{N K}}\right.}{|V|}$.

Proof. For steady state agent injection, the probability that an agent will not visit road segment $i$ in successive $K$ hops is $\left(1-\pi_{i}\right)^{K}$. Let $\theta(N, K)$ be the set containing road segments that are visited by at least one agent. The expected cardinality of $\theta(N, K)$ thus can be obtained as:

$|\theta(N, K)|=\sum_{i \in V} 1-\left(1-\pi_{i}\right)^{N \times K}$.

Since $\Gamma(N, K)=\frac{|\theta(N, K)|}{|V|}$, we have:

$\Gamma(N, K)=\frac{\sum_{i \in V} 1-\left(1-\frac{d_{i}}{2|E|}\right)^{N K}}{|V|}$

One problem with Lemma 1 is that it requires the target area to be first converted into a finite undirected graph, and then solved for the steady state probabilities for random walk. We could eliminate this requirement if the graph was regular, i.e., each node having the identical degree. Interestingly, studies have shown that most road networks are highly regular [42]. For regular road networks, Lemma 1 thus can be further simplified as:

Lemma 2. Under the assumption of saturated traffic and steady state agent injections, for regular road networks, $\Gamma(N, K)=1-\left(1-\frac{1}{|V|}\right)^{N K}$.

Proof. According to the Handshake Lemma in Graph Theory, $\sum_{i \in V} d_{i}=2|E|$. Thus, for a regular graph, we have $\frac{d_{i}}{2|E|}=\frac{1}{|V|}$. By replacing $\frac{d_{i}}{2|E|}$ by $\frac{1}{|V|}$ in equation (4), we have $\Gamma(N, K)=$ $1-\left(1-\frac{1}{|V|}\right)^{N K}$.

From Lemma 1 and 2, we obtain the following results:

1. The expected coverage reaches its limiting value of $100 \%$ as the number of agents increases. Arbitrarily high coverage can be achieved by simply injecting a large number of agents.

2. The expected coverage has a diminishing return with increasing $N$. Therefore, to increase expected coverage beyond a very high value, such as $95 \%$, may require too many more agents to be injected. 


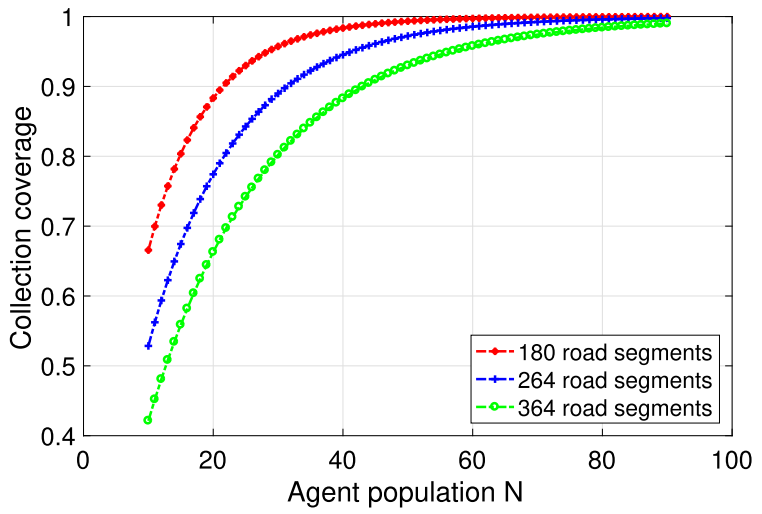

Fig. 6. Numerical results for coverage as a function of agent population under saturated traffic.

For $K=20$, Fig. 6 shows numerical results for three different sizes of grid road networks containing a total of 180,264 , and 364 road segments, respectively. As we can see from the figure, irrespective of the size of the road network, the coverage asymptotically reaches $100 \%$ of the target area as we increase the number of agents. The diminishing return is also clear. Due to the diminishing return, it may be desirable to achieve a target coverage, such as $95 \%$ of the total road segments, instead of injecting a massive number of agents for a small improvement in coverage.

It is also intuitive to see that for a given target coverage, larger road networks require more agents. From Fig. 6 we observe that for a given target coverage, the ratio between agent population to the total number of road segments in the road network maintains at the same level. This observation inspires us to further investigate the relationship between agent population and the size of the road network.

Definition 2. Agent density, $\lambda=\frac{N}{|V|}$, is defined as the ratio of total number of agents injected to the total number of road segments in the target road network.

Theorem 1. Under the assumption of saturated traffic and steady state agent injections, for large regular road networks, the required agent density $\lambda$ for a target coverage, $C_{\text {target }}$, is independent of road network size, which can be approximated by the constant $-\ln \left(1-C_{\text {target }}\right) / K$.

Proof. From Lemma 2, we have $C_{\text {target }}=1-\left(1-\frac{1}{|V|}\right)^{N K}$. Taking logarithm on both sides, we obtain:

$\ln \left(1-C_{\text {target }}\right)=N K \ln \left(1-\frac{1}{|V|}\right)$

Since, $\ln (1-x) \approx-x$ if $\mathrm{x} \approx 0$, for large $|V|$, we have:

$\frac{N}{|V|} \approx-\ln \left(1-C_{\text {target }}\right) / K$

Theorem 1 formally shows that $\lambda$ only depends on the target coverage $C_{\text {target }}$ and is inversely proportional to $K$. Fig. 7 shows the required agent density to achieve different target coverages as a function of the total number of road segments of the target area for $K=20$. We can see that the agent density is relatively constant for a target coverage value. For example, to achieve 99\% coverage, the system needs to inject only 0.2 agents per road segment, or only one agent per 5 road segments, irrespective of the size of the target area.

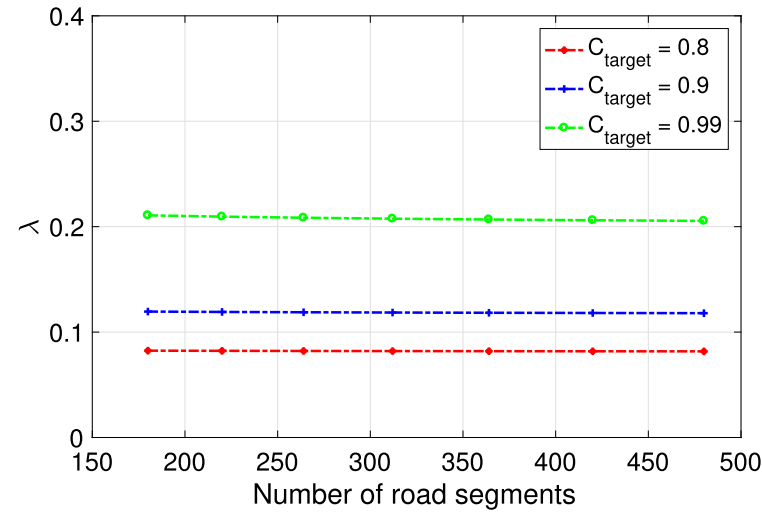

Fig. 7. Numeric results for the required agent density as a function of target area size under saturated traffic

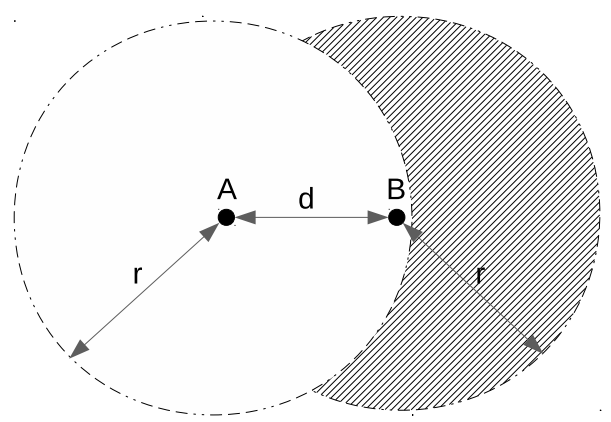

Fig. 8. Area covered by two consecutive agent broadcasts.

\subsection{Derivation of $\Omega(t)$}

Suppose that time is slotted and each agent hops at each time slot $\Delta t$. Let us assume that the geographical area of the target urban region is $D$ and the total number of vehicles in the area is $M$. Each vehicle has a DSRC transmission range of $r$.

Lemma 3. The probability $\operatorname{Pr}(t)$ that a vehicle overhears a circulating agent after time $t$ is $1-(1-\delta) \times(1-0.41 \delta)^{t}$, where $\delta=\frac{\pi r^{2}}{D}$ represents the fraction of the target area covered by DSRC radio range.

Proof. Consider the scenario in Fig. 8, where an agent is forwarded from vehicle $A$ to $B$ using a DSRC broadcast. Since the agent migration and rebroadcast by $B$ take only a few milliseconds, it is reasonable to assume that the locations of vehicles do not change significantly. The unshaded circle is the area covered by the broadcast from $A$, while the shaded region represents the new area covered by the rebroadcast from $B$. Using basic geometry, the area of the shaded region can be obtained as:

$\mathcal{A}(d)=\pi r^{2}-4 \int_{d / 2}^{r} \sqrt{r^{2}-x^{2}} \mathrm{~d} x$

where $d$ denotes the distance between $A$ and $B$.

Since $d$ can vary from a very small value close to zero to the maximum value equals to the DSRC communications range $r$, the average new area, $\alpha$, covered by each hop excluding the area covered in the previous hop can be obtained $\mathrm{as}^{1}$ :

\footnotetext{
1 The approximation of $0.41 \pi r^{2}$ can be obtained by integrating equation (7) ove the circle of radius $x$ centred at vehicle $A$ for $x$ in $[0, . r]$
} 
$\alpha=\int_{0}^{r} \frac{2 \pi x \times \mathcal{A}(x)}{\pi r^{2}} \mathrm{~d} x \approx 0.41 \pi r^{2}$

The expected number of vehicles in the shaded area then can be derived as $\frac{\alpha M}{D}$. If we let $C(t)$ denote the expected number of vehicles overhearing a given agent after time $t$, then we can obtain the fraction of all vehicles in $D$ that did not overhear the agent as $1-\frac{C(t)}{M}$. Therefore, during each time slot $\Delta t$, the expected number of new vehicles covered by an agent is simply $\frac{\alpha M}{D}\left(1-\frac{C(t)}{M}\right)$, which is basically the rate at which an agent can cover new vehicles in the target area. Therefore, we have the following relationships:

$\left\{\begin{array}{l}C(t)-C(t-1)=\frac{\alpha M}{D}\left(1-\frac{C(t-1)}{M}\right) \\ C(0)=\pi r^{2} \frac{M}{D}\end{array}\right.$

Here, $C(0)$ refers to the expected number of vehicles overhearing a given agent at the beginning of the dissemination process. Equation (9) is therefore a linear non-homogeneous recurrence with initial value of $C(0)$. By solving the recurrence, we obtain:

$C(t)=M-\left(M-\pi r^{2} \frac{M}{D}\right) \times\left(1-\frac{\alpha}{D}\right)^{t}$

Now the probability $\operatorname{Pr}(t)$ that a vehicle will overhear the agent after time $t$ can be calculated as:

$\operatorname{Pr}(t)=\frac{C(t)}{M}=1-(1-\delta) \times(1-0.41 \delta)^{t}$

Lemma 3 shows that under the assumption of saturated traffic, $\operatorname{Pr}(t)$ is independent of the vehicle density and only depends on $\delta$, i.e., the fraction of the geographical area $D$ covered by DSRC communication range $r$.

Now assuming that there are $N$ agents migrating among vehicles independently, the expected number of agents that a vehicle overhears after time $t$ is simply obtained as $N \times \operatorname{Pr}(t)$. Recall that Lemma 1 gives the expected fraction of road segments that can be learned after receiving $N$ agents. Therefore the dissemination speed $\Omega(t)$ can be calculated by simply replacing $N$ in equation (4) with $N \times \operatorname{Pr}(t)$.

Fact 1. Under the assumption of saturated traffic, for arbitrary road network and $N$ agents, the dissemination speed satisfies $\Omega(t)=1-$ $\frac{\sum_{i \in V}\left(1-\pi_{i}\right)^{K N P r(t)}}{|V|}$.

Fact 2. Under the assumption of saturated traffic, for regular road networks and $N$ agents, $\Omega(t)=1-\left(1-\frac{1}{|V|}\right)^{K N P r(t)}$.

Fig. 9 shows numerical results of $\Omega(t)$ for $N=20 / 40 / 60$ for the grid road network with 180 road segments, where the area of the region is $5.0625 \mathrm{~km}^{2}$ and the DSRC communication range is set to $250 \mathrm{~m}$. It is clear that the expected fraction of road segments a vehicle learns asymptotically reaches a certain value. In fact, this limiting value is defined by $\Gamma(N, K)$ as the maximum vehicle awareness cannot exceed the collection coverage. On the other hand, each curve also shows a clear diminishing return, which indicates that vehicles can build their knowledge very quickly in the beginning, but the rate of learning decreases dramatically after some period of time. For example, in the case of 60 agents, a vehicle is expected to learn around $90 \%$ of the road segments by the 50th time step, but it then takes another 50 time steps to learn the rest of road segments. In another word, after a certain threshold, agents should terminate themselves as the benefit of continuing dissemination becomes negligible.

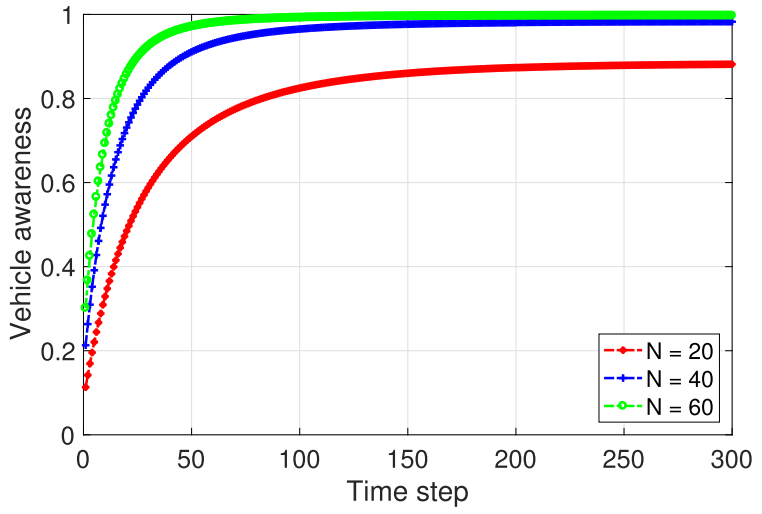

Fig. 9. Numeric results for dissemination speed in the grid containing 180 road segments under saturated traffic.

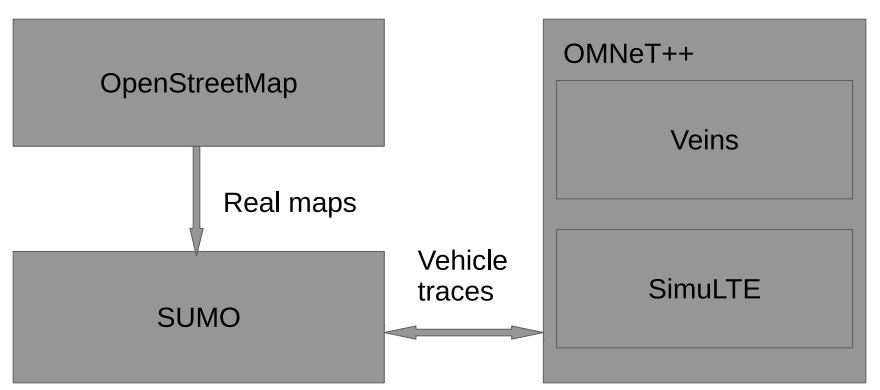

Fig. 10. Multi-layer simulation platform.

\section{Simulation}

In the previous section, we derived analytical models of the proposed CarAgent system by simplifying the physical environment wherever necessary for mathematical tractability. Using state-ofthe-art simulation tools, in this section, we study the effect of many real-world parameters that were ignored by the analytical models. These simulation experiments thus give us the opportunity to not only validate the efficacy of the analytical models but also gain insights into a range of other performance issues not captured by the models. The simulation tools and experimental setup are described first, followed by the simulation results.

\subsection{Simulation setup}

We implemented the proposed CarAgent system in a multilayer simulation platform as shown in Fig. 10. The simulation platform includes three interconnected tools, OpenStreetMap, SUMO, and OMNet++. OpenStreetMap is used for obtaining real-world maps including detailed information of the road network, traffic light and buildings. This information is then imported to SUMO [43] to generate realistic vehicle traces using microscopic vehicular mobility models. OMNet++, which includes simuLte [44] and Veins modules [45], is bidirectionally coupled with SUMO providing wireless network simulation. Veins provides a complete set of models for assessing DSRC vehicular networks, while simulte is responsible for LTE communication networks.

In the analytical models, we ignored the actual topology of the roads and approximated the road networks as regular graphs. However, in real life, the actual road topologies vary from cities to cities, and they are not exactly regular, i.e., every road segment does not have identical degree or connectivity. In our simulation, we therefore simulate road networks with different topologies and different levels of regularity. We also consider varying sizes of target areas for data collection and dissemination. In particular, we choose road networks from three different cities in the world, 


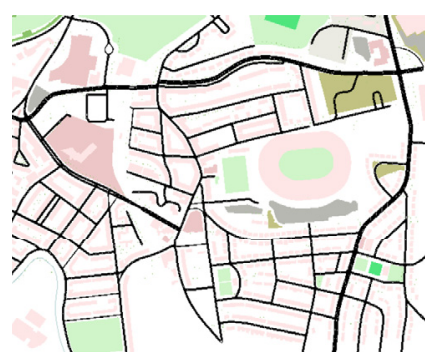

(a) Glasgow, UK

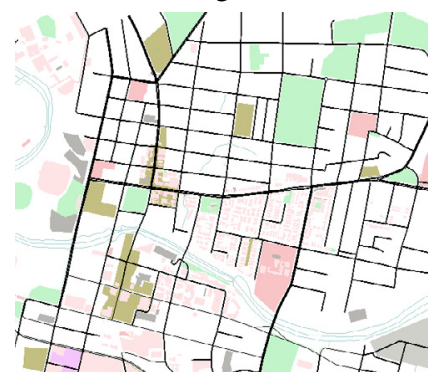

(c) Sydney, Australia

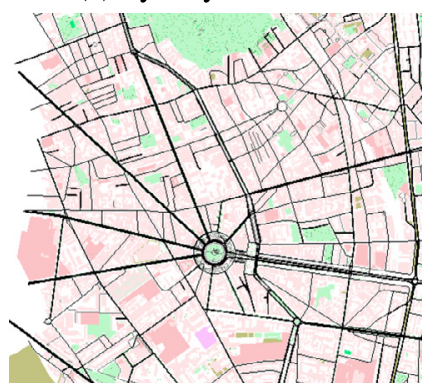

(e) Paris, France

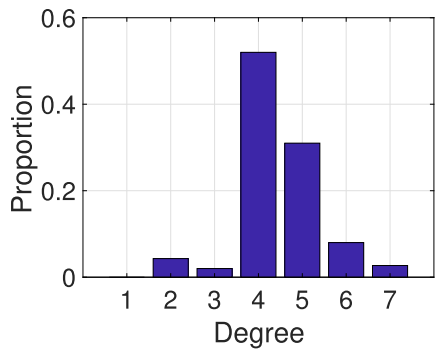

(b) Glasgow, UK

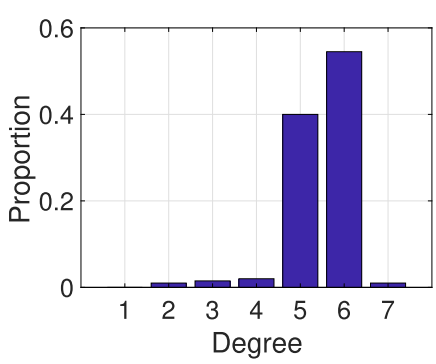

(d) Sydney, Australia

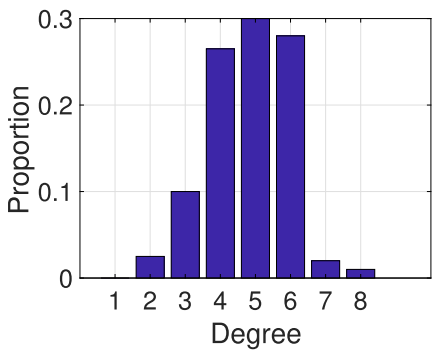

(f) Paris, France
Fig. 11. The urban maps and their degree distributions.

Table 1

Road network statistics.

\begin{tabular}{llll}
\hline Parameter & Glasgow & Sydney & Paris \\
\hline Area $\left(\mathrm{km}^{2}\right)$ & 5.15 & 9.34 & 10.08 \\
Total number of road segments & 204 & 328 & 410 \\
Total length of roads $(\mathrm{km})$ & 27.27 & 50.84 & 66.74 \\
Mean of degree & 4.863 & 5.4850 & 4.4450 \\
Standard deviation of degree & 0.9745 & 0.8210 & 1.1419 \\
\hline
\end{tabular}

Glasgow, Sydney, and Paris, which fulfil our objective of creating variations in topology, regularity, and size. Transport literature [42] categorises all road network into two basic groups, grid and radial. We therefore, consider one grid network (Sydney), one radial (Paris), and one hybrid (Glasgow). The simulated maps, as extracted from OpenStreetMap, are shown in Fig. 11 along with their respective degree distributions. We observe that Glasgow and Sydney have a high level of regularity (lower spread of degree), while Paris is less regular (wider spread of degree). Table 1 summarises the main statistics of these three road networks and shows that Paris network has twice the size of Glasgow network in terms of area, number of road segments, and the total length of roads.

For mathematical tractability, we completely ignored the mobility details of the cars in our analytical models. In this section, we simulate the following details to capture the realistic movement of cars on our roads. All cars enter the road network from random locations with an initial velocity randomly chosen between $40-60 \mathrm{~km} / \mathrm{h}$. Each vehicle chooses randomly a pair of starting lo-
Table 2

Simulation parameters.

\begin{tabular}{lll}
\hline Type & Parameter & Value \\
\hline Scenario & Maps & Sydney/Paris/Glasgow \\
& Vehicle density & 4 to 50 vehs/km \\
& Maximum speed & $60 \mathrm{~km} / \mathrm{hour}$ \\
& & \\
& Bit rate & $6 \mathrm{Mbps}$ \\
& Bandwidth & $10 \mathrm{MHz}$ \\
& Frequency band & $5.9 \mathrm{GHz}$ \\
& MAC MTU & $2312 \mathrm{bytes}$ \\
& MAC + PHY header & $450 \mathrm{bits}$ \\
& Tx Power & $20 \mathrm{~mW}$ \\
LTE & Propagation model & Nakagami-m \\
& eNodeB Tx Power & $46 \mathrm{dBm}$ \\
& UE Tx Power & $20 \mathrm{dBm}$ \\
& Propagation model & Jakefading \\
& Number of RBs (UL/Dl) & $50 / 50$ \\
& Network + transport header & $48 \mathrm{bytes}$ \\
& Number of eNodeBs & $5 / 9 / 10$ \\
& & \\
CarAgent & Aggregate size & $100 \mathrm{bytes}$ \\
& $K$ & 20 \\
& $t_{\text {wait }}$ & $25 \mathrm{~ms}$ \\
\hline
\end{tabular}

cation and destination, where the route is computed in advance by using the standard Dijkstra algorithm. Note that even though the traffic demands are modelled by using random trips, the resulting vehicle density is non-uniform at different parts of the road network. This is because during the simulation, the vehicle's mobility is defined by the default carFollowing-Krauss model, including stopping at traffic lights, which ultimately breaks the uniformity. We define traffic density as the number of vehicles per $\mathrm{km}$ and control the average density for a simulated road network of a given total road length by adjusting the total vehicles injected into the network.

The rest of the simulation parameters are summarised in Table 2. A noteworthy detail not considered in our analytical study is the radio propagation model. In our simulation, all DSRC communications employ the Nakagami-m fading with $m=3$, plus the SimpleObstacleShadowing model in Veins to mimic the signal shadowing effect due to buildings in the urban region, where building details are provided by the OpenStreetMap. To enable LTE communications, we place an LTE eNodeB (base station) for each square $\mathrm{km}$ in each map.

Agents perform local data aggregation after each hop using the following generic aggregation scheme: individual FCDs from the same road segment are merged into a single FCD aggregate. We assume the size of execution code and the size of each FCD aggregate is 100 bytes, which means that a maximum of 20 such aggregates can be carried by a single DSRC MAC frame given the Maximum Transfer Unit (MTU) for DSRC is 2312 bytes. As a result, we assume $K=20$. Each round of simulation starts with a $200 \mathrm{~s}$ of warmup time, and then a number of agents are injected into the road network. These agents first collect and upload FCD aggregates, and then roam inside the VANET for another $T_{d s}=10 \mathrm{~s}$ for dissemination purpose. Every simulation is repeated 10 times with independent random number seeds and the 95\% confidence interval has been reported for each collected performance metric.

In addition to the two primary metrics, Collection Coverage and Dissemination Speed, defined in Section 4, we also collect the following statistics from our simulations:

- Duplicate ratio: It is the fraction of uploaded aggregates that are duplicates.

- Aggregation factor: It is defined as the number of individual FCDs that are aggregated into a single aggregate, which provides a measure of aggregation efficiency. 


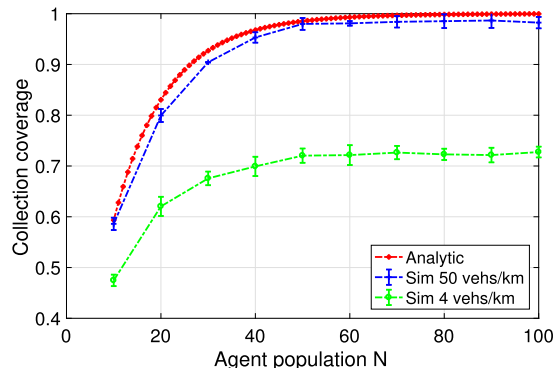

(a) Glasgow

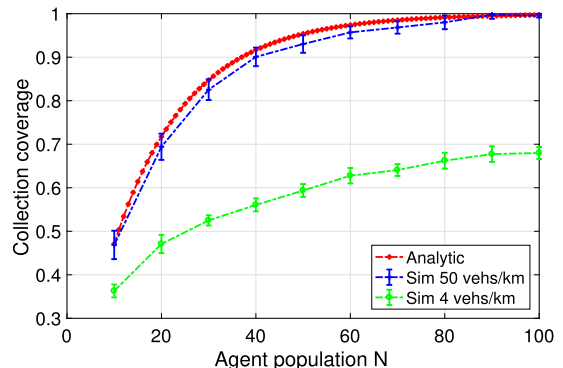

(b) Sydney

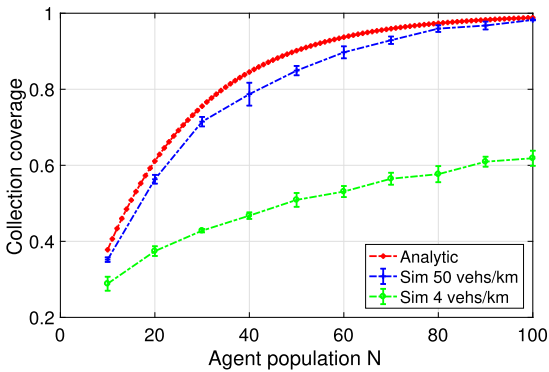

(c) Paris

Fig. 12. Collection coverage as a function of agent population $N$.

- LTE cost: It is measured by both the number of bytes uploaded via the LTE uplink and the number of Resource Blocks (RBs) consumed at the base stations for transmitting these data. We assume each uploaded LTE message consists of 48 bytes of network and transport headers and a payload consisting of the collected aggregates by an agent.

- DSRC cost: It is measured by the total number of bytes being broadcast over the DSRC channel during the given simulation time, excluding the number of bytes being broadcast due to CAMs exchange.

\subsection{Collection coverage}

For all three cities, Fig. 12 shows the collection coverage results obtained as a function of the number of agents, where the analytic results are obtained using Lemma 2 under the assumption of regular road networks and saturated traffic. For the simulation results, we consider both dense traffic with enough vehicles on the road (50 vehs $/ \mathrm{km}$ ) as well as unusually light traffic (4 vehs $/ \mathrm{km}$ ). We make the following observations.

The analytical model of Lemma 2 serves as an upper-bound, which is achieved when roads are very regular and there are enough vehicles on the road for the agents to hop in all directions. For example, for all three cities, we can see a very good match between analytical and simulation results for $50 \mathrm{vehs} / \mathrm{km}$. The match for Paris is only slightly worse compared to Glasgow and Sydney due to having a slightly less regular road network. Note that the good alignment between analytical and simulation results are obtained irrespective of road topologies, i.e., grid vs. radial. On the other hand, collection coverage obtained from simulation is way below the analytical predictions when only $4 \mathrm{vehs} / \mathrm{km}$ is simulated. This is because the agents cannot always find vehicles in all directions, hence hopping back and forth between the same road segments limiting the potential to cover more road segments during the 20 hops.

From Fig. 12, we can deduce that the analytical model is more sensitive to the vehicle density compared to the topology or regularity of the road networks. The question we therefore must answer is: what is the threshold for vehicle density for which Lemma 2 provides accurate predictions? In order to answer this question, we conducted another set of simulations by varying traffic density from 4 to 50 vehs $/ \mathrm{km}$ for a target coverage of $99 \%$ (agent density $\lambda=0.2$ ). The results presented in Fig. 13 show that close to $99 \%$ target in collection coverage can be achieved for all three cities for vehicle density greater than 20 vehs $/ \mathrm{km}$. The fact that all three cities achieve the same target coverage of $99 \%$ despite having different network sizes further validates Theorem 1, which stipulates that a given agent density will realise a specific target coverage irrespective of the size of the road network.

Note that the threshold of 20 vehs/ $\mathrm{km}$ is very common in urban regions. For example, the authors in [46] show that the traffic vol-

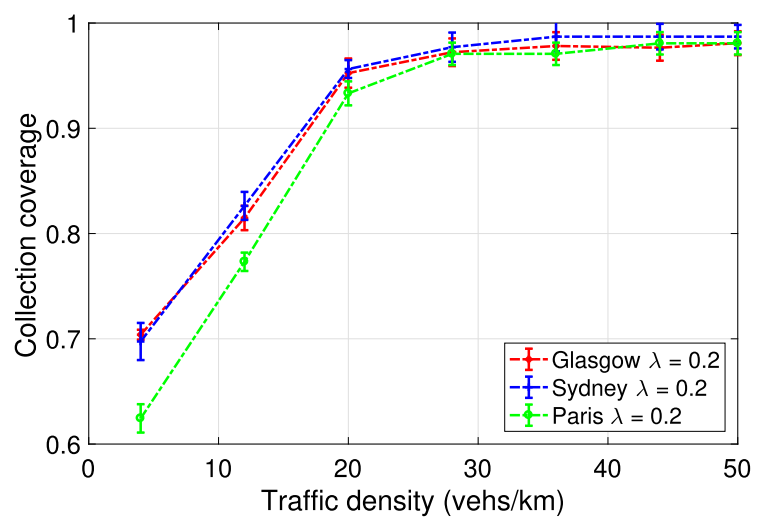

Fig. 13. Collection coverage as a function of traffic density for $\lambda=0.2$.

Table 3

Duplicate ratio for three maps $(\lambda=0.2)$.

\begin{tabular}{|c|c|c|c|c|c|c|}
\hline \multirow[b]{2}{*}{ Traffic density (vehs/km) } & \multicolumn{2}{|c|}{ Glasgow } & \multicolumn{2}{|c|}{ Sydney } & \multicolumn{2}{|l|}{ Paris } \\
\hline & 20 & 50 & 20 & 50 & 20 & 50 \\
\hline Distinct segments & 14.95 & 15.03 & 14.37 & 14.93 & 15.02 & 15.17 \\
\hline Stored aggregates & 5.91 & 5.96 & 6.02 & 5.94 & 5.90 & 5.86 \\
\hline Duplicate ratio & 0.153 & 0.145 & 0.143 & 0.146 & 0.152 & 0.149 \\
\hline
\end{tabular}

ume on all road types in Sydney urban region would be higher than 800 vehs/hour from $6 \mathrm{AM}$ to $21 \mathrm{PM}$, which translates to a traffic density of 20 vehs $/ \mathrm{km}$ if the average vehicle speed is $40 \mathrm{~km} /$ hour. Similarly, the real traffic data presented in [47] shows that the average traffic density in the road networks for Beijing is higher than 20 vehs/km for more than 16 hours per day.

\subsection{Reduction of duplicate uploads}

Table 3 shows the performance of CarAgent in eliminating duplicate uploads over LTE. We observe that most of the duplicates are successfully eliminated before uploading. For example, the third column shows that after completing 20 hops, each agent collects around 15 distinct road segment aggregates, but only around 5.9 of them on average are stored and uploaded, indicating that most of the duplicates are successfully detected to save LTE cost. However, as CarAgent avoids centralised control, a small percentage of duplicates, about $15 \%$ (last column), still goes undetected. Nevertheless, as will be shown later in this section, CarAgent significantly outperforms the state-of-the-art (OFC) in terms of reducing the LTE cost.

\subsection{LTE cost}

We then assess LTE cost by comparing the proposed CarAgent system with the state-of-the-art, the On-the-Fly Clustering (OFC) 


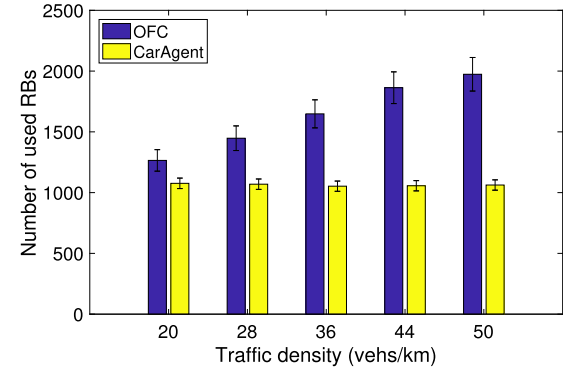

(a) Glasgow

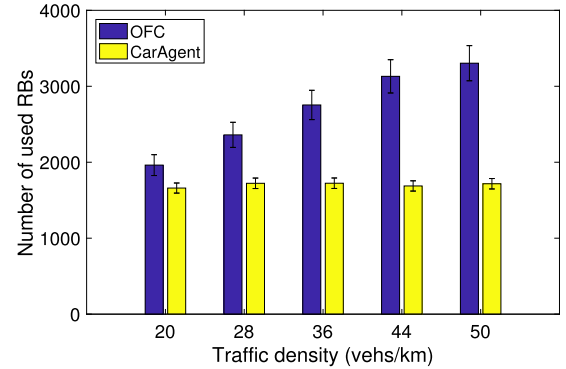

(b) Sydney

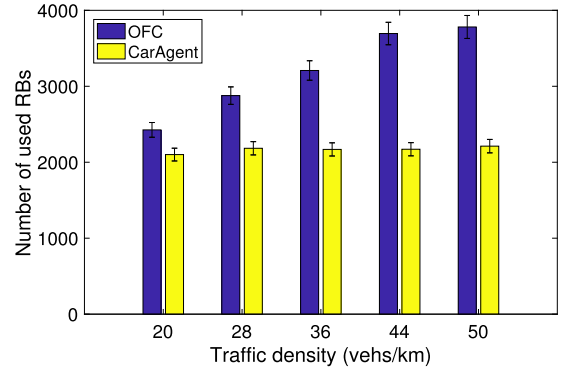

(c) Paris

Fig. 14. Comparison of OFC and CarAgent in terms of RB usage in LTE base stations.

Table 4

Aggregation factor comparison of OFC and CarAgent.

\begin{tabular}{lllllll}
\hline & \multicolumn{2}{l}{ 20 vehs $/ \mathrm{km}$} & & & 50 vehs $/ \mathrm{km}$ & \\
\cline { 2 - 3 } \cline { 6 - 7 } \cline { 6 - 7 } & CarAgent & OFC & & CarAgent & OFC \\
\hline Collection coverage & $96.92 \%$ & $97.58 \%$ & & $98.02 \%$ & $98.74 \%$ \\
Aggregation factor & 4.50 & 2.69 & & 9.43 & 4.78 \\
Number of bytes uploaded & 39914.57 & 49730.77 & & 39078.73 & 76497.33 \\
DSRC packet collisions per second & 3885.68 & 4021.32 & & 26483.52 & 27032.33 \\
\hline
\end{tabular}

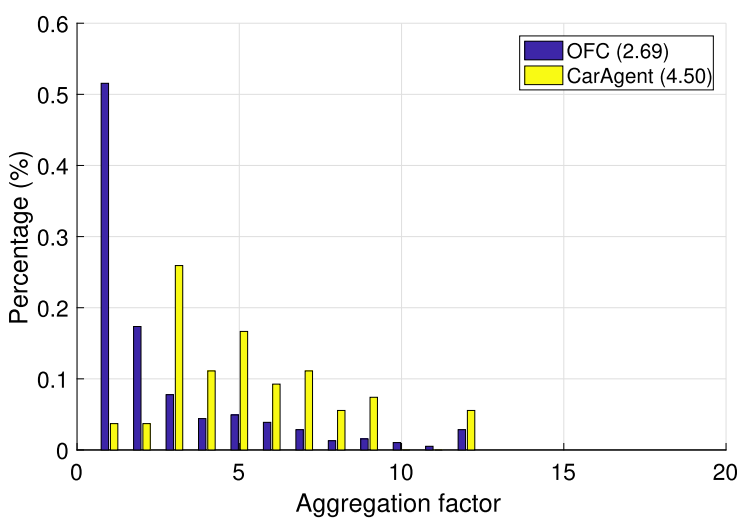

(a) $20 \mathrm{vehs} / \mathrm{km}$

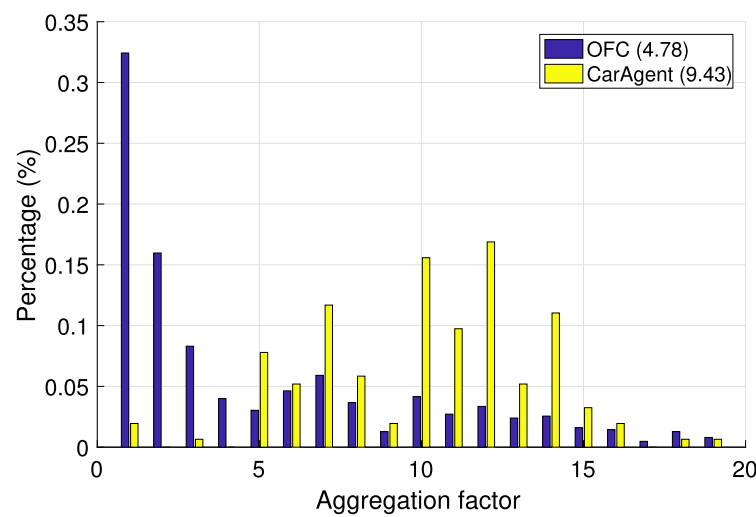

(b) $50 \mathrm{vehs} / \mathrm{km}$

Fig. 15. PMF of aggregation ratio for OFC and CarAgent.

approach in [32]. Briefly speaking, the OFC approach employs a contention process based on the current LTE channel quality and the number of one hop DSRC neighbours for cluster head elections, such that vehicles whose timer first expire become the cluster head and broadcasting an "inhibit" message to suppress others within the DSRC communication range. Since the original OFC performs exhaustive FCD collection, to conduct a fair comparison, we implemented a modified version that assumes cluster heads also perform local data aggregation before uploading, using the same aggregation scheme used in CarAgent (i.e. individual FCDs from the same road segment are merged into a single FCD aggregate). The rest of parameters remain the same with the original settings.

As explained earlier, LTE cost for data collection can be quantified by both the RB consumptions in the base stations as well as the total number of bytes uploaded. For increasing traffic density on the road, RB usage and bytes transferred are compared for OFC and CarAgent in Fig. 14 and Table 4, respectively. We observe that $\mathrm{RB}$ usage remains constant for CarAgent despite the increase in traffic density. This is because CarAgent produces a single aggregate (100 bytes) for every road segment that has at least one car. Therefore, as long as traffic is saturated, i.e., at least one car every road segment, we have the same amount data produced irrespective of the vehicle density.
In contrast, increasing vehicular traffic also increases wireless traffic (e.g. CAMs exchange), which results in increasing number of packet collisions due to hidden terminals as shown in Table 4. As a result, the contention process is less reliable in dense traffic, and a larger number of clusters are formed, because vehicles might not be able to overhear the "inhibit" message even they are located at the proximity. This directly lead to a lower aggregation efficiency (lower aggregation factor) and a higher number of aggregates per road segment uploaded to the cloud. Fig. 15 plots the probability mass function (PMF) of the aggregation factor of uploaded FCD aggregates for the OFC and the CarAgent. We observe that in the case of the OFC, the aggregation factor of more than $80 \%$ of aggregates is less than 3; for traffic density of $20 \mathrm{vehs} / \mathrm{km}$, around $50 \%$ of uploaded aggregates are actually individual FCDs. The situation worsens for 50 vehs $/ \mathrm{km}$. Consequently, both RB usage and total bytes transferred increase with increasing traffic density. For 50 vehs $/ \mathrm{km}$, CarAgent consumes 50\% less LTE resources compared to OFC.

\subsection{Dissemination speed}

In the following sections, we evaluate the dissemination performance of the CarAgent by comparing it with the ATB approach in $[18,19]$. Same as their original work, each vehicle maintains a 


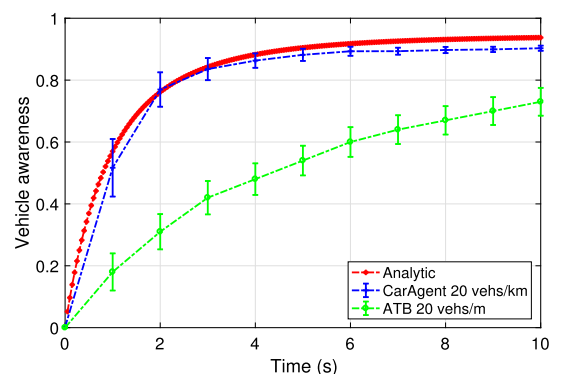

(a) Glasgow

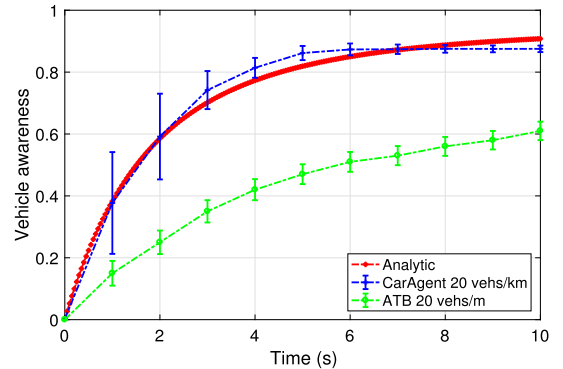

(b) Sydney

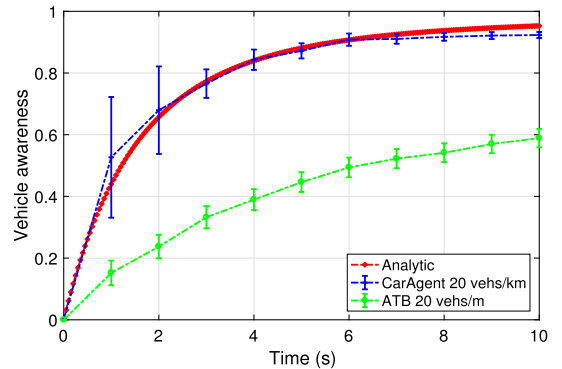

(c) Paris

Fig. 16. Dissemination speed.

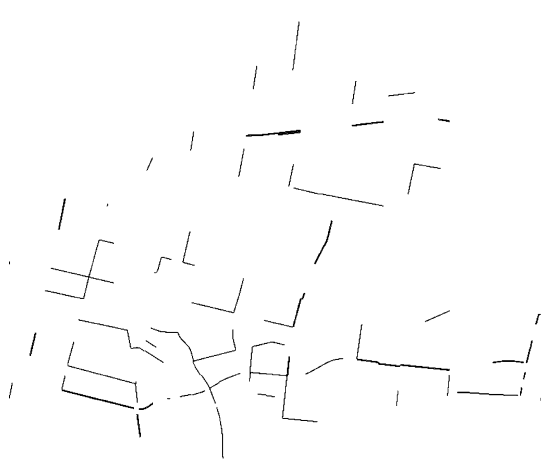

(a) $t=2 s$

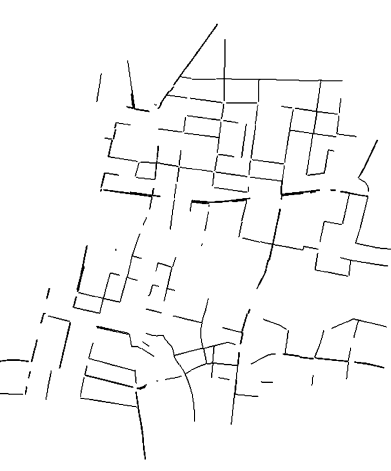

(b) $t=4 s$

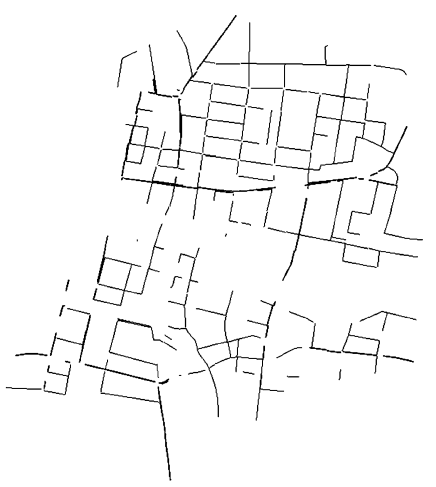

(c) $\mathrm{t}=10 \mathrm{~s}$

Fig. 17. Isoline maps of the known road segments.

local knowledge base storing only the most recent FCD aggregate for each road segment, and each ATB broadcast frame contains at most 20 entries randomly selected from the list.

For vehicle density of $20 \mathrm{vehs} / \mathrm{km}$ and agent density of 0.2 (target collection coverage of 99\%), Fig. 16 plots the percentage of road segments known by any vehicle on average for both CarAgent and ATB as a function of elapsed time. For CarAgent we plot both the analytical results obtained from Fact 2 and the simulation results. We observe that the analytical model can accurately predict dissemination speed for all three cities under $20 \mathrm{vehs} / \mathrm{km}$. As predicted by Fact 2, the rate at which vehicles learn new road segments diminishes as time progresses, which is further illustrated by plotting the isoline map of the known road segments in Fig. 17 after 2, 4, and 10 seconds. As we can see, during the first 2 seconds, the vehicle only knows a small proportion of road segments sampled randomly around the region. The awareness then grows quickly after 4 seconds, covering almost the whole region. The rate of learning new road segments then starts to slow down. After 10 seconds, the vehicles only learn a few additional road segments.

We then compare CarAgent with the ATB. For CarAgent, we still set $\lambda=0.2$ (e.g. target coverage $=99 \%$ ). From Fig. 16, we observe that with CarAgent, vehicles can learn the traffic conditions in the target area much faster than if they used the ATB approach. For example, after 5 seconds, vehicles learn $80 \%$ of the target area compared to only $50 \%$ with ATB. Similarly, with CarAgent, it takes only 2 seconds to learn $60 \%$ of the area while it takes 10 seconds for ATB. The improved dissemination speed in CarAgent can be explained by the high speed at which the agents cover the region by hopping from cars to cars, while the ATB approach relies on the mobility of the cars to spread the information, which is limited by the moving speed of the cars.

\subsection{DSRC overhead of FCD dissemination}

Next, we investigate the DSRC overhead of CarAgent and compare it against ATB. To focus on the DSRC overhead caused by FCD aggregation dissemination only, we exclude the number bytes broadcast over the DSRC channel due to CAMs exchange and only count the traffic due to the CarAgent and the ATB. For increasing traffic density, Fig. 18 compares the DSRC cost for CarAgent and ATB. We observe that with the adaptive adjustment of broadcast interval, the DSRC cost of ATB only increases slightly with the increase of vehicular density. However, CarAgent utilises even fewer channel resources irrespective of the vehicular density. For FCD dissemination, CarAgent can reduce DSRC cost by around $45 \%$. The success of CarAgent is attributed to the fact that DSRC is only used by a finite number of agents, which is independent of traffic density, and these agents almost do not store any duplicated information among each others.

\section{Conclusion}

In this work, we propose CarAgent, an integrated FCD collection and dissemination framework. CarAgent is based on a hybrid architecture involving both DSRC and LTE, where the LTE network is used for data collection, while the dissemination operation can be off-loaded to the local DSRC. Through analytic models and extensive simulations, we have demonstrated that CarAgent is truly scalable offering only limited communication overhead for both LTE and DSRC, which is independent of the vehicular traffic density. Compared to the state-of-the-art, CarAgent reduces LTE resource consumption by $50 \%$ and DSRC channel utilisation by $45 \%$. Our results have also verified that the proposed CarAgent can work effectively over different road network patterns ranging from the simple grid to complex patterns involving both grid and radial networks. By adjusting the number of agents, the server can achieve 


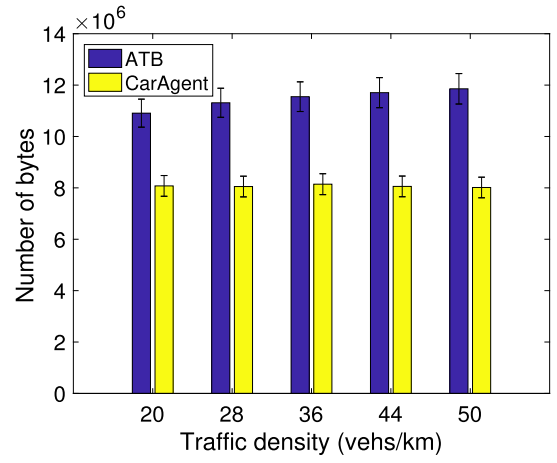

(a) Glasgow

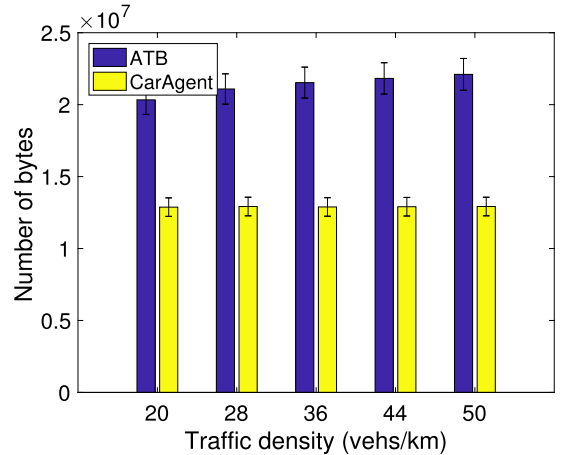

(b) Sydney

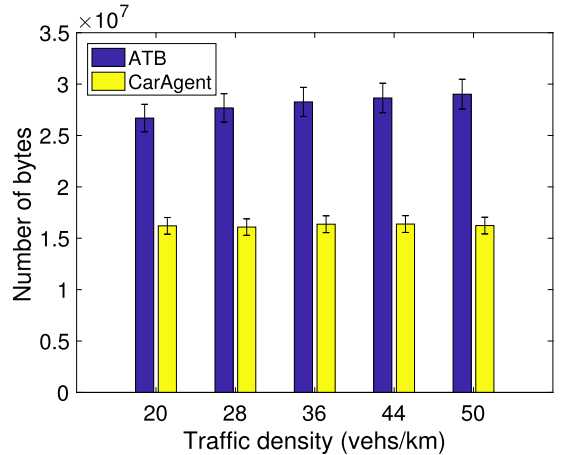

(c) Paris

Fig. 18. Total number of bytes broadcast on the DSRC channel as a function of vehicle density.

close to $100 \%$ collection coverage while vehicles can learn the collected FCD through DSRC within a few seconds.

\section{References}

[1] R. Quintero, A. Llamazares, D. Llorca, M. Sotelo, L. Bellot, O. Marcos, I. Daza, C. Fernández, Extended floating car data system-experimental study, in: Proc. of the IEEE Intelligent Vehicles Symposium (IV), 2011, pp. 631-636.

2] P. Salvo, I. Turcanu, F. Cuomo, A. Baiocchi, I. Rubin, Heterogeneous cellular and DSRC networking for floating car data collection in urban areas, Veh. Commun. 8 (2017) 21-34.

[3] J.E. Naranjo Hernández, F. Jiménez Alonso, F. Serradilla García, J.G. Zato Recellado, Comparison between floating car data and infrastructure sensors for traffic speed estimation, in: Proc. of the IEEE International Conference on Intelligent Transportation Systems (ITSC), 2010.

[4] F. Rempe, P. Franeck, U. Fastenrath, K. Bogenberger, Online freeway traffic estimation with real floating car data, in: Proc. of the IEEE International Conference on Intelligent Transportation Systems (ITSC), 2016, pp. 1838-1843.

[5] R. Blokpoel, J. Vreeswijk, Uses of probe vehicle data in traffic light control, Transp. Res. Proc. 14 (2016) 4572-4581.

[6] J.B. Kenney, Dedicated short-range communications (DSRC) standards in the United States, Proc. IEEE 99 (7) (2012) 1162-1182.

[7] ETSI, 302665 v1. 1.1: Intelligent Transport Systems (ITS), communications architecture, European Standard (Telecommunications Series).

[8] K. Zheng, Q. Zheng, P. Chatzimisios, W. Xiang, Y. Zhou, Heterogeneous vehicular networking: a survey on architecture, challenges, and solutions, IEEE Commun. Surv. Tutor. 17 (4) (2015) 2377-2396.

[9] C. Ide, F. Kurtz, C. Wietfeld, Cluster-based vehicular data collection for efficient LTE machine-type communication, in: Proc. of the IEEE Vehicular Technology Conference (VTC Fall), 2013, pp. 1-5.

[10] S. Ucar, S.C. Ergen, O. Ozkasap, Multihop-cluster-based IEEE 802.11 p and LTE hybrid architecture for VANET safety message dissemination, IEEE Trans. Veh. Technol. 65 (4) (2016) 2621-2636.

[11] P.M. D’Orey, N. Maslekar, I. de la Iglesia, N.K. Zahariev, NAVI: neighbor-aware virtual infrastructure for information collection and dissemination in vehicular networks, in: Proc. of the Vehicular Technology Conference (VTC Spring), 2015.

[12] G. Remy, S.-M. Senouci, F. Jan, Y. Gourhant, LTE4V2X: LTE for a centralized VANET organization, in: Proc. of the IEEE Global Telecommunications Conference (GLOBECOM), 2011, pp. 1-6.

[13] G. Rémy, S.-M. Senouci, F. Jan, Y. Gourhant, LTE4V2X - collection, dissemination and multi-hop forwarding, in: Proc. of the IEEE Conference on Communications (ICC), 2012, pp. 120-125.

[14] S. Dietzel, J. Petit, F. Kargl, B. Scheuermann, In-network aggregation for vehicular ad hoc networks, IEEE Commun. Surv. Tutor. 16 (4) (2014) 1909-1932.

[15] R. Du, C. Chen, B. Yang, N. Lu, X. Guan, X. Shen, Effective urban traffic monitoring by vehicular sensor networks, IEEE Trans. Veh. Technol. 64 (1) (2015) 273-286.

[16] K. Abboud, W. Zhuang, Stochastic modeling of single-hop cluster stability in vehicular ad hoc networks, IEEE Trans. Veh. Technol. 65 (1) (2016) 226-240.

[17] T. Nadeem, P. Shankar, L. Iftode, A comparative study of data dissemination models for VANETs, in: Proc. of the IEEE Annual International Conference on Mobile and Ubiquitous Systems-Workshops, 2006, pp. 1-10.

[18] C. Sommer, O.K. Tonguz, F. Dressler, Traffic information systems: efficient message dissemination via adaptive beaconing, IEEE Commun. Mag. 49 (5) (2011).

[19] K.Z. Ghafoor, K.A. Bakar, M. van Eenennaam, R.H. Khokhar, A.J. Gonzalez, A fuzzy logic approach to beaconing for vehicular ad hoc networks, Telecommun. Syst. 52 (1) (2013) 139-149.

[20] G. Bansal, J.B. Kenney, Controlling congestion in safety-message transmissions: a philosophy for vehicular DSRC systems, IEEE Veh. Technol. Mag. 8 (4) (2013) 20-26.
21] W. Klein Wolterink, G. Heijenk, H. van den Berg, Analytically modelling the performance of piggybacking on beacons in VANETs, in: Proc. of the ACM International Workshop on Vehicular Inter-networking, Systems, and Applications, 2012, pp. 43-52.

[22] M. Chen, S. Gonzalez, Y. Zhang, V.C. Leung, Multi-agent Itinerary Planning for Wireless Sensor Networks, Quality of Service in Heterogeneous Networks, 2009, pp. 584-597.

[23] C. Konstantopoulos, A. Mpitziopoulos, D. Gavalas, G. Pantziou, Effective determination of mobile agent itineraries for data aggregation on sensor networks, IEEE Trans. Knowl. Data Eng. 22 (12) (2010) 1679-1693.

[24] A. Kanan, T. Eldos, M. Al-Kahtani, Mobile ad hoc networks routing using ant colony optimization, World Comput. Sci. Inf. Technol. J. 3 (6) (2013).

[25] D. Kadono, T. Izumi, F. Ooshita, H. Kakugawa, T. Masuzawa, An ant colony optimization routing based on robustness for ad hoc networks with GPSs, Ad Hoc Netw. 8 (1) (2010) 63-76.

[26] G. Singh, N. Kumar, A.K. Verma, Ant colony algorithms in MANETs: a review, IEEE J. Netw. Comput. Appl. 35 (6) (2012) 1964-1972.

[27] S.J. Gudakahriz, S. Jamali, E. Zeinali, NISR: a nature inspired scalable routing protocol for mobile ad hoc networks, J. Comput. Sci. Eng. Technol. 1 (4) (2011) $180-184$.

[28] A. Soua, H. Afifi, Adaptive data collection protocol using reinforcement learning for VANETs, in: Proc. of the IEEE International Wireless Communications and Mobile Computing Conference (IWCMC), 2013, pp. 1040-1045.

[29] R.T. Meier, J. Dunkel, Y. Kakuda, T. Ohta, Mobile agents for service discovery in ad hoc networks, in: Proc. of the IEEE International Conference on Advanced Information Networking and Applications (AINA), 2008, pp. 114-121.

[30] T. Hashimoto, J. Aoki, T. Ohta, Y. Kakuda, Performance evaluation of mobile agent-based service collection and dissemination scheme considering entrance and exit of nodes in VANETs, in: Proc. of the IEEE International Symposium on Computing and Networking (CANDAR), 2014, pp. 284-287.

[31] F. Dressler, H. Hartenstein, O. Altintas, O. Tonguz, Inter-vehicle communication quo vadis, IEEE Commun. Mag. 52 (6) (2014) 170-177.

[32] I. Turcanu, C. Sommer, A. Baiocchi, F. Dressler, Pick the right guy: CQI-based LTE forwarder selection in VANETs, in: Proc. of the IEEE Vehicular Networking Conference (VNC), 2016, pp. 1-8.

[33] S. Panichpapiboon, W. Pattara-Atikom, A review of information dissemination protocols for vehicular ad hoc networks, IEEE Commun. Surv. Tutor. 14 (3) (2012) 784-798.

[34] M. Chaqfeh, A. Lakas, I. Jawhar, A survey on data dissemination in vehicular ad hoc networks, Veh. Commun. 1 (4) (2014) 214-225.

[35] ETSI, 102 962, Intelligent transport systems (ITS), Framework for public mobile networks in cooperative ITS (C-ITS) (Tech. Rep. No. 1.1. 1). ETSI Technical Committee Intelligent Transport System. European Telecommunications Standards Institute (2012)

[36] H. Füßler, J. Widmer, M. Käsemann, M. Mauve, H. Hartenstein, Contentionbased forwarding for mobile ad hoc networks, Ad Hoc Netw. 1 (4) (2003) 351-369.

[37] F. Klingler, F. Dressler, C. Sommer, IEEE 802.11 p unicast considered harmful, in: Proc. of the IEEE Vehicular Networking Conference (VNC), 2015, pp. 76-83.

[38] S. Kuhlmorgen, I. Llatser, A. Festag, G. Fettweis, Performance evaluation of ETS geonetworking for vehicular ad hoc networks, in: Proc. of the IEEE Vehicular Technology Conference (VTC Spring), 2015.

[39] S. Kuhlmorgen, A. Festag, G. Fettweis, Impact of decentralized congestion control on contention-based forwarding in VANETs, in: Proc. of the IEEE World of Wireless, Mobile and Multimedia Networks (WoWMoM), 2016, pp. 1-7.

[40] ETSI, 302 636-4-1 v1.2.1: Intelligent Transport Systems (ITS); Vehicular Communications, European Standard (Telecommunications Series).

[41] L. Rodero-Merino, A.F. Anta, L. López, V. Cholvi, Performance of random walks in one-hop replication networks, Comput. Netw. 54 (5) (2010) 781-796.

[42] Y. Duan, F. Lu, Robustness of city road networks at different granularities, Phys. A, Stat. Mech. Appl. 411 (2014) 21-34. 
[43] M. Behrisch, L. Bieker, J. Erdmann, D. Krajzewicz, SUMO-simulation of urban mobility: an overview, in: Proc. of the Third International Conference on Advances in System Simulation, 2011, pp. 63-68.

[44] A. Virdis, G. Stea, G. Nardini, SimuLTE-a modular system-level simulator fo LTE/LTE-A networks based on OMNeT++, in: Proc. of the IEEE International Conference on Simulation and Modeling Methodologies, Technologies and Applications (SIMULTECH), 2014, pp. 59-70.

[45] C. Sommer, R. German, F. Dressler, Bidirectionally coupled network and road traffic simulation for improved IVC analysis, IEEE Trans. Mob. Comput. 10 (1) (2011) 3-15.
[46] N. Orthongthed, B. Wang, J. Legaspi, Estimating cost expansion factors in the Sydney urban and NSW rural road networks for economic evaluation of road projects, in: Australasian Transport Research Forum (ATRF), 2013.

[47] B. Jing, L. Wu, H. Mao, S. Gong, J. He, C. Zou, G. Song, X. Li, Z. Wu, Development of a vehicle emission inventory with high temporal-spatial resolution based on NRT traffic data and its impact on air pollution in Beijing - Part 1: development and evaluation of vehicle emission inventory, Atmos. Chem. Phys. 16 (5) (2016) 3161-3170. 